\title{
A Planning-oriented Resilience Assessment Framework for Transmission Systems under Typhoon Disasters
}

Liu, Xiaonan; Hou, Kai; Jia, Hongjie; Zhao, Junbo; Mili, Lamine; Jin, Xiaolong; Wang, Dan

Published in:

IEEE Transactions on Smart Grid

Link to article, DOI:

10.1109/TSG.2020.3008228

Publication date:

2020

Document Version

Peer reviewed version

Link back to DTU Orbit

Citation (APA):

Liu, X., Hou, K., Jia, H., Zhao, J., Mili, L., Jin, X., \& Wang, D. (2020). A Planning-oriented Resilience

Assessment Framework for Transmission Systems under Typhoon Disasters. IEEE Transactions on Smart Grid, 11(6), 5431 - 5441. https://doi.org/10.1109/TSG.2020.3008228

\section{General rights}

Copyright and moral rights for the publications made accessible in the public portal are retained by the authors and/or other copyright owners and it is a condition of accessing publications that users recognise and abide by the legal requirements associated with these rights.

- Users may download and print one copy of any publication from the public portal for the purpose of private study or research.

- You may not further distribute the material or use it for any profit-making activity or commercial gain

- You may freely distribute the URL identifying the publication in the public portal 


\title{
A Planning-oriented Resilience Assessment Framework for Transmission Systems under Typhoon Disasters
}

\author{
Xiaonan Liu, Kai Hou, Member, IEEE, Hongjie Jia, Senior Member, IEEE, Junbo Zhao, Senior Member, IEEE, \\ Lamine Mili, Life Fellow, IEEE, Xiaolong Jin, Member, IEEE, Dan Wang, Member, IEEE
}

\begin{abstract}
In this paper, a planning-oriented resilience assessment framework is proposed that can help to design a more resilient power transmission system against extreme weather-induced events, such as typhoon disasters. It consists of three major models, i) a wind field-based probabilistic typhoon model to determine the level of uncertainty in the intensity and path of the typhoon; ii) a spatiotemporal fragility model of the power transmission corridor to quantify the spatiotemporal impacts of extreme wind speed on the failure probability of transmission corridor, and iii) a resilience assessment model of the planning system that allows us to develop quantitative resilience indices from both the system and component perspectives. The combinatorial enumeration method is introduced into the proposed framework to generate various potential typhoon disasters. Meanwhile, the impact-incrementbased state enumeration (IISE) method is embedded within the proposed framework to improve its computational efficiency. The proposed method can provide the key information to identify the weak points of the system as well as inform the design of enhanced resilient planning schemes of transmission system against typhoon disasters. Extensive simulations carried out on the IEEE RTS-79 system considering realistic typhoon scenarios demonstrate the effectiveness of the proposed method.
\end{abstract}

Index Terms-Resilience assessment, spatiotemporal fragility model, planning, probabilistic typhoon model, impact-increment

\section{INTRODUCTION}

$\mathrm{T}$ he continuous development of long-distance and largecapability power transmission system has resulted in an unprecedented growth in their scale and complexity. A number of quantities of long-distance transmission corridors including transmission lines and towers may be exposed to harsh environmental conditions for a long period of time, increasing their vulnerabilities to extreme weather conditions. In the light of global climate change, extreme weather events, such as

This work was supported by the National Natural Science Foundation of Chin (Grant No.51707129, Grant No. U1766210, Grant No. 51977140, Grant No. 51977141, Grant No. 5191101844) and the Natural Science Foundation of Tianjin City (Grant No. 18JCQNJC07600). Junbo Zhao and Lamine Mili are supported partly by the US National Science Foundation under grant ECCS-1917308.

Xiaonan Liu, Kai Hou, Hongjie Jia and Dan Wang are with Key Laboratory of Smart Grid of Ministry of Education, and Tianjin Key Laboratory of Smart Energy and Information Technology, Tianjin University, Tianjin, 300072, China. (e-mail: xiaonanliu@tju.edu.cn, hdbhyj@tju.edu.cn, hjjia@tju.edu.cn, wangdantjuee@tju.edu.cn).

Junbo Zhao is with the Department of Electrical and Computer Engineering, Mississippi State University, Starkville, MS, 39762, USA (email: junbo@ece.msstate.edu).

Lamine Mili is with the Bradley Department of Electrical and Compute Engineering, Virginia Polytechnic Institute and State University, Northern Virginia Center, Falls Church, VA 22043, USA, (email: lmili@vt.edu).

Xiaolong Jin is with the Center for Electric Power and Energy (CEE), Department of Electrical Engineering, Technical University of Denmark (DTU) 2800 Kgs. Lyngby, Denmark (e-mail: xiajin@elektro.dtu.dk). typhoons, are becoming recurrent, resulting in an unprecedentedly large number of power outages all around the world [1]-[4]. Despite this fact, the impacts of extreme weather conditions are often ignored during the planning of power transmission systems

To overcome this problem, research on power system resilience to natural disasters has gained popularity in the past few years [5]-[9]. According to the outcomes of such papers, there is a necessity for seeking an effective way to capture the adverse impacts of possible adverse weather incidences on transmission systems within the planning period. Gaining such information is essential in enhancing system resilience during the planning stage.

There are a number of extreme weather events that could temper with the power transmission system. This paper, however, focuses specifically on typhoons. When a typhoon occurs, the super-intense wind speed normally leads to a surge in the failure rates and failure probabilities of the transmission components. Such failures often result in severe load shedding. It is, therefore, necessary to construct the wind field model of the typhoon to predict the wind speed at different geographical locations. For this purpose, many scholars have studied the wind filed model of typhoons for various application scenarios. Batts et al., [10], for instance, used an empirical formula to establish a wind field model considering the attenuation after typhoon landing. Shapiro, [11], on the other hand, developed a wind field model by solving the momentum equation of atmospheric motion. Meng et al. [12] established an analytical wind field model based on the perturbation equilibrium equation. The wind speed, at any point in the typhoon wind field, can be obtained by the above-mentioned models. However, typhoons usually occur randomly in the planning prospect, thereby making the existing models fail to capture their probabilistic features. As a result, it is difficult to make longterm predictions considering various potential typhoon disasters within the planning period aiming at initiating precaution measures.

The challenge of incorporating grievous weather impacts in planning system resilience assessment is about revealing the relationship between the failure probability of each transmission component and the wind speed. To solve it, Liu et al. [13] and Li et al. [14] adopted the fuzzy cluster method to obtain the nonlinear relationship between wind speed and the failure rate of the transmission lines. Liu et al. [15] and Han et al. [16], on the other hand, used statistical regression methods 
to predict the wind speed related to power outages during the dreadful weather. Despite their general plausibility, these models only provide a certain failure probability of the transmission components under typhoons. They do not well capture the impact of continuous change of wind speed over time. To overcome these shortcomings, Panteli et al. [17]-[19] constructed a fragility curve by determining the failure probability of the transmission component via a function of wind speed. Wang et al. [20] and Li et al. [21] show that the failure rate of components is related to the square of wind speed. Due to the lack of spatial and temporal typhoon model, the transmission system is usually assumed to be exposed to the same wind speed in the aforementioned literature. However, a practical transmission system generally covers a large geographic area. Consequently, different transmission corridors, or even different segments of the same corridor, may suffer from different wind speeds when the typhoon moves across. Therefore, it is necessary to build a model that can reflect the temporal and spatial failure probability of different transmission corridors when a typhoon moves across with attenuation.

Regarding the resilience indices, most of the existing ones are based on the resilience triangle, which is defined as the integral of the system performance degradation and the degradation time [22]. Panteli et al. [23] extended the resilience triangle into a multi-phase resilience trapezoid. The resilience trapezoid can further quantify how fast the system performance is dropped when a severer weather incident struck a system and how fast the system recovers to its previous normal state. Regarding the transmission system, the load demand is usually used to depict system performance. Espinoza et al. [24] and Amirioun et al. [25] quantified the system resilience as a function of the amount of load demand that is not provided due to the catastrophic effects of extreme weather conditions. In this regard, a smaller amount of load shedding depicts higher system resilience. Johnson et al., [26] developed the Demand Not Served (DNS) index by using non-sequential Monte Carlo simulation (MCS) methods to assess power system resilience. Amirioun et al. [9] introduced a vulnerability index to evaluate the level of system performance degradation. That index can provide a quick perception of how the system performance is impacted by the extreme weather events. However, these indices can only reflect the resilience from the view of the whole system. They cannot aid planners to identify specific vulnerability cases, such as the vulnerable transmission corridors. By analyzing the impacts of various potential typhoon disasters on each transmission corridor, the resilience of the planning system can be comprehensively investigated, and the weak points of the system can be fully determined. This allows planners to optimize the resilience enhancement strategy of the transmission system. Therefore, it is of urgent need to build a model that can not only assess whether the system is resilience enough against typhoon disasters, but also determine its weak points.

To sum up, this paper aims at developing a planning-oriented resilience assessment framework to assess the resilience of the power system against typhoon disasters and determine its weak points. By analyzing the impacts of various potential typhoon disasters on each transmission corridor, the resilience of the power system can be comprehensively investigated, and the weak points of the system can be fully determined. In addition, the framework can further guide the resilience enhancement measures to strengthen the weak points, such as expanding or upgrading the vulnerable transmission corridors. The proposed framework includes three major parts:

1) A probabilistic typhoon model is proposed by analyzing the key parameters of the wind field model and their probability distributions. This allows us to simulate various potential typhoon disasters within the planning time.

2) A spatiotemporal fragility model of a transmission corridor, subdivided into a combination of a series of transmission line segments and transmission towers, is developed to investigate the cumulative spatiotemporal effect of the typhoon duration on the transmission corridor failure probability. This couples the impacts of typhoon disaster and power transmission systems.

3) A resilience assessment model of the planning transmission power system is proposed from both the system and the component perspectives. This provides the planner with the key information needed to identify the weak points of the system as well as to inform about the design of enhanced resilient planning schemes.

It is worth noting that the proposed framework is also suitable for power system resilience assessment under other types of extreme weather events. The rest of the paper is organized as follows. Section II introduces the basic theory of resilience assessment. The proposed resilience assessment framework and its algorithm implementation are elaborated in Section III. Numerical studies are presented in Section IV, and conclusions are drawn in Section V.

\section{BASIC THEORY OF RESILIENCE ASSESSMENT}

\section{A. Wind Field Model of Typhoon}

Batts model is a mature wind field model that is simple and fast to estimate [10]. It is used in this paper to simulate the realtime wind speed at different locations of the affected areas. This model assumes that the attenuation of the typhoon over time is caused by the reduction of the pressure difference between the center and the periphery of the typhoon.

The attenuation model of the pressure difference at a certain time $t$ when the typhoon travels over land is expressed as

$$
\Delta H(t)=\Delta H_{0}-0.02[1+\sin (\varphi-\theta)] t,
$$

where $\triangle H(t)$ is the central pressure difference at time $t$ (hour), $\triangle H_{0}$ is the original central pressure difference before the typhoon landing, they are given in inch, $\theta$ is the angle between the typhoon motion direction and the due north direction - the clockwise is positive, $\varphi$ is the angle between coastline and the due north direction.

Based on the original central pressure difference, the maximum gradient wind speed is expressed as

$$
v_{g x}(t)=K \sqrt{\Delta H(t)},
$$


where $K$ is a coefficient, typically set between $6.97\left(\mathrm{~m} / \mathrm{s} / \mathrm{mm}^{1 / 2}\right)$ at latitude $23^{\circ} \mathrm{N}$ to $6.93\left(\mathrm{~m} / \mathrm{s} / \mathrm{mm}^{1 / 2}\right)$ at latitude $45^{\circ} \mathrm{N}$ [10]. And, the central pressure difference in (2) is given in $\mathrm{mm}$.

Then the maximum wind speed is obtained by

$$
v_{r_{\max }}(t)=0.865 v_{g t}(t)+0.5 v_{T} \text {, }
$$

where $v_{T}$ is the translational speed of typhoon $(\mathrm{m} / \mathrm{s})$. Generally, the path of the typhoon motion is assumed to be a straight line.

The wind speed of a certain location in the typhoon wind field is given by [27]

$$
v_{r}(t)=\left\{\begin{array}{ll}
v_{r_{\max }}(t) d(t) / r_{\text {max }}(t) & , d(t) \leq r_{\text {max }} \\
v_{r_{\max }}(t)\left(r_{\text {max }}(t) / d(t)\right)^{0.6} & , d(t)>r_{\text {max }}
\end{array},\right.
$$

where $d(t)$ is the distance between a location and the typhoon center at time $t$, and $r_{\max }$ is the radius of maximum wind speed, which can be obtained by [28]-[29]

$$
r_{\max }(t)=\exp \left(2.63-5.086 \times 10^{-5} \Delta H(t)^{2}+0.0395 y(t)\right),
$$

where $y(t)$ is the latitude of typhoon center at time $t$. It should be noted that the $\triangle H$ in (5) is given in $\mathrm{mb}$.

\section{B. Resilience Assessment Indices for Transmission System}

Quantitative indices are necessary to assess and enhance the resilience of transmission system against typhoon disasters. The resilience triangle provides an effective way to quantify the resilience of the system, especially in the planning stage [22]. As shown in Fig. 1, the performance curve of a normal system can be described by $Q_{0}$, while the performance curve during the typhoon affecting period can be described by $Q(t)$. $Q_{1}$ represents the worst degraded performance level. With the resilience triangle, the resilience index of the system can be defined as the normalized area of the performance degradation during the period of typhoon occurrence. Therefore, the quantitative resilience index can be obtained by $\Re$. A higher index corresponds to lower system resilience.

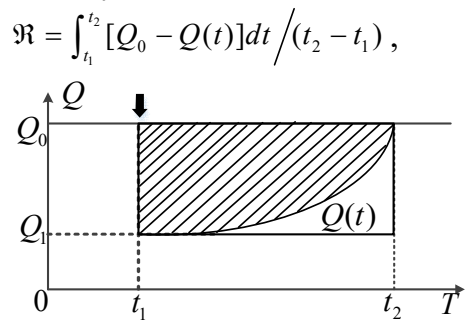

Fig. 1. The resilience triangle and rectangle.

It is noticeable that the above index can only assess the resilience of the transmission system caused by a certain disturbance. Since the transmission system may face different intensities of typhoons, it is necessary to consider the resilience under various potential typhoon disasters during the planning stage. In this paper, we have modified the resilience index to comprehensively reflect the expected system performance degradation induced by various scenarios of typhoons. It is worth noting that the failure scenarios are considered as a stable state after various remedial action schemes have been taken. Another downside of that index lies in its lack of consideration of the uncertainty of typhoons. Normally, typhoon disasters are different, and quantifying them uniformly is not accurate. Only the most serious performance degradation is considered, and the resilience triangle has been simplified into a resilience rectangle. To consider typhoon uncertainty, the index is modified as

$$
R=E[\Re] \approx E\left[Q_{0}-Q_{1}\right]=\sum_{w \in W} P_{w}\left(Q_{0}-Q_{1}\right)_{w}=\sum_{w \in W} P_{w} Q_{w},
$$

where $E[]$ is the expected value of the resilience index $\Re, Q_{w}$ is the worst performance degradation incurred by a certain typhoon $w, P_{w}$ is the probability of typhoon $w$, and $W$ is the set of various potential typhoons in the affected area. In this paper, load shedding is used to quantify the performance degradation. Their values can be obtained from the AC optimal power flow.

\section{Proposed Planning-ORIENTEd RESILIENCE ASSESSMENT FRAMEWORK}

The proposed resilience assessment framework is shown in Fig. 2. It consists of the following three main components: 1) a probabilistic typhoon model that presents the uncertainty of typhoon during the planning period 2) a spatiotemporal fragility model of the transmission corridors to characterize their failure probabilities as a function of the wind speed and 3) a resilience assessment model to obtain the resilient indices for both the system and its components.

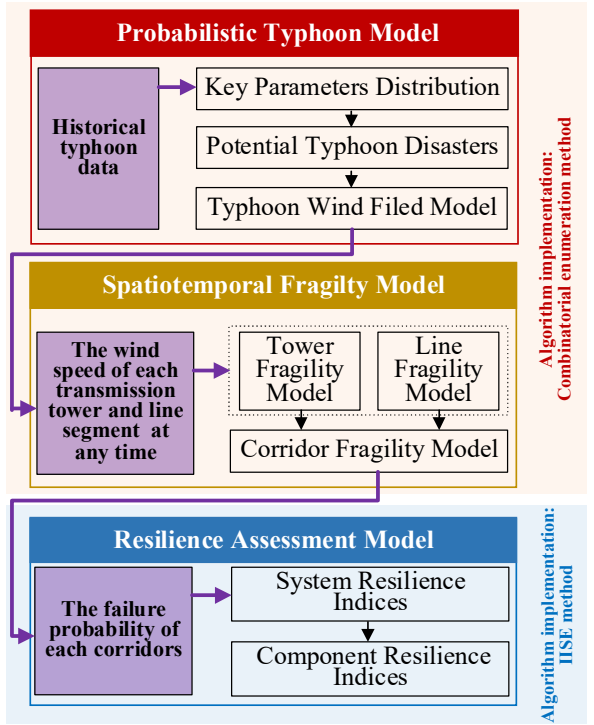

Fig. 2. The proposed resilience assessment framework.

\section{A. Probabilistic Typhoon Model}

Evaluating the comprehensive spatiotemporal impacts of typhoons on the transmission systems is possible under the premise that the occurrence of a typhoon in a certain region follows a stochastic model. Identifying these key parameters of this model and their probability distributions allows us to quantify the uncertainty of the occurrence of a typhoon. As shown in Fig. 3, the green circles represent the radius of maximum wind speed at different times. The green dotted line represents the path of the typhoon motion. The orange shade indicates the affected rang of the typhoon and the wind speed. The darker the color, the higher the wind speed is. Due to the attenuation of the typhoons, the wind speed at a given transmission corridor varies with time. 
Generally, the unit of pressure difference given by meteorological department is hectoPascal pressure $(\mathrm{hPa})$. However, the central pressure difference $\triangle H(t)$ is given in inch in (1) and in $\mathrm{mm}$ in (2), so (1) needs to be transformed into the following form:

$$
\Delta H^{\prime}(t)=0.75 \Delta H_{0}-0.508[1+\sin (\varphi-\theta)] t,
$$

where the $\triangle H^{\prime}(t)$ is given in $\mathrm{mm}$.

Substituting (8) into (3) and considering the maximum wind speed at time $t, v r_{\max }(t)$ can be obtained by

$$
\begin{array}{r}
\left.v_{r_{\max }}(t)=6.029 \sqrt{0.75 \Delta H_{0}-0.508[1+\sin (\varphi-\theta) t}\right]+0.5 v_{T}, \\
T=1.476 \Delta H_{0}[1+\sin (\varphi-\theta)](9)
\end{array}
$$

By translating the latitude and longitude coordinates to the rectangular axes, the coordinate of the typhoon center at time $t$ can be obtained by

$$
\begin{aligned}
& x(t)=x_{0}+v_{T} t \sin (-\theta+\pi / 2)=x_{0}+v_{T} t \sin \theta, \\
& \mathrm{y}(t)=y_{0}+v_{T} t \cos (-\theta+\pi / 2)=y_{0}+v_{T} t \sin \theta,
\end{aligned}
$$

Thus, the distance between a location and the typhoon center at time $t$ can be obtained by

$$
d(t)=\sqrt{\left[x_{d}-\left(x_{0}+v_{T} t \sin \theta\right)\right]^{2}+\left[y_{d}-\left(y_{0}+v_{T} t \cos \theta\right)\right]^{2}},
$$

where $x_{d}$ and $y_{d}$ are the longitude and the latitude of $d$ meters from the typhoon center, respectively; $x_{0}$ and $y_{0}$ are the longitude and the latitude of the typhoon landing sites, respectively. In addition, the duration time of typhoon $T$ is related to the central pressure difference and can be obtained by

$$
T=1.476 \Delta H_{0}[1+\sin (\varphi-\theta)],
$$

In Fig. $3, d\left(t_{1}\right)$ and $d\left(t_{2}\right)$ represent the distance of this line segment from the typhoon center at times $t_{1}$ and $t_{2}$, respectively. $v_{r}\left(t_{1}\right)$ and $v_{r}\left(t_{2}\right)$ represent the wind speeds at times $t_{1}$ and $t_{2}$, respectively. $r_{\max }\left(t_{1}\right)$ and $r_{\max }\left(t_{2}\right)$ represent the radius of maximum wind speed at times $t_{1}$ and $t_{2}$, respectively. $v r_{\max }\left(t_{1}\right)$ and $v r_{\max }\left(t_{2}\right)$ are the maximum wind speeds at times $t_{1}$ and $t_{2}$, respectively.

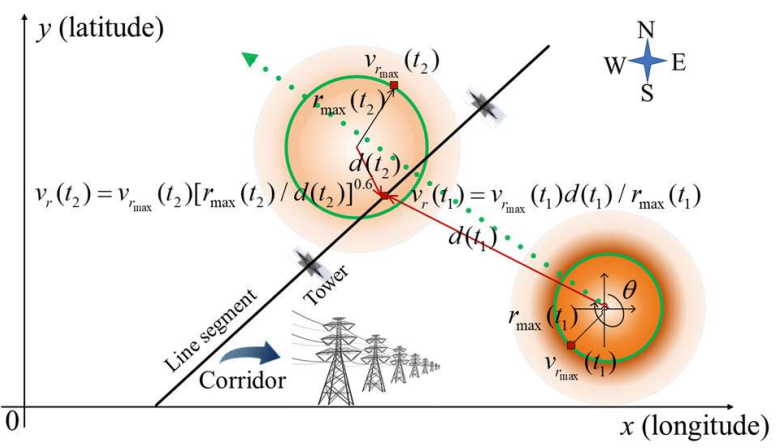

Fig. 3. The moving process of a certain typhoon.

Key parameters that directly affect the wind speed at any location of a typhoon can be further inferred from (9) and (12): the original pressure difference $\Delta H_{0}$, the translational speed $v_{T}$, the motion direction $\theta$, and the longitude and the latitude of typhoon landing site $\left(x_{0}, y_{0}\right)$. Based on the statistical information of the historical typhoon disasters in a certain region, the probability distribution can be estimated for such parameters. Thus, a large number of potential typhoons and their occurrence probabilities can be generated by sampling or enumeration from the probability density functions of those key parameters.

\section{B. Spatiotemporal Fragility Model of a Transmission Corridor}

A transmission corridor comprises a set of transmission lines and towers, which are generally more likely to be subject to the damages of typhoons. On the contrary, transformers and cables are almost impervious to extreme wind speed [5]. Therefore, we only focus on the typhoon-induced failures of lines and towers when analyzing the typhoon-induced impacts on transmission systems. Transmission corridors are usually very long, so the wind speed varies in different corridor segments. In this paper, a long transmission corridor is equivalent to a series of transmission line segments that are connected via two adjacent transmission towers, as shown in Fig. 3. Consequently, the equivalent failure probability of a transmission corridor can be modeled as a series system with several line segments and towers.

To quantitatively assess the resilience of a transmission system under typhoon disasters, the core work is to establish the relationship between the wind speed and the failure probability of each transmission corridor from both temporal and spatial perspectives. In this regard, a spatiotemporal fragility model is developed when the typhoon moves across the transmission system. Using this model ensures that its cumulative failure probability within the typhoon duration $T$ can be obtained.

The failure rate $\lambda_{m, k}(t)$ of the $k$ th tower of the $m$ th transmission corridor at time $t_{i}$ can be expressed as follows:

$$
\lambda_{m, k}\left(t_{i}\right)=\left\{\begin{array}{cc}
0, & v_{m, k}\left(t_{i}\right) \in\left[0, v_{d, \text { tower }}\right] \\
e^{\gamma\left[v_{m, k}\left(t_{i}\right)-2 v_{d, \text { tower } r}\right]}, & v_{m, k}\left(t_{i}\right) \in\left[v_{d, \text { tower }}, 2 v_{d, \text { tower }}\right], \\
1, & v_{m, k}\left(t_{i}\right) \in\left[v_{e x}, \infty\right]
\end{array}\right.
$$

where $v_{m, k}\left(t_{i}\right)$ is the real-time wind speed of the $k$ th tower of the $m$ th transmission corridor at time $t_{i}, \gamma$ is the model coefficient, considered to be 0.2 in our model [30], and $v_{d, \text { tower }}$ is the design wind speed of each tower, considered to be $35 \mathrm{~m} / \mathrm{s}$ in our model [30].

Since the wind speed at different locations cannot be directly expressed as a continuous function of time, the total typhoon duration $T$ can be divided into several shorter periods based on the integral theory. The wind speed and the failure rate at each shorter period are then obtained as a constant. Then, the cumulative failure probability of the $k$ th tower of the $m$ th transmission corridor in the period $T$ is expressed as

$$
\begin{aligned}
p_{m, k} & =1-\exp \left\{-\int_{0}^{T}\left[\lambda_{m, k} /\left(1-\lambda_{m, k}\right)\right]\right\} d t \\
& =1-\exp \left\{-\sum_{i=0}^{N-1} \int_{t_{i}}^{t_{i}+\Delta t}\left[\lambda_{m, k}\left(t_{i}\right) /\left(1-\lambda_{m, k}\left(t_{i}\right)\right)\right] d t\right\}, \\
& =1-\exp \left\{-\sum_{i=0}^{N-1}\left[\lambda_{m, k}\left(t_{i}\right) /\left(1-\lambda_{m, k}\left(t_{i}\right)\right)\right] \Delta t\right\}
\end{aligned}
$$

where $N$ is the total number of shorter period, and $\triangle t$ is the time interval of each shorter period.

The line segment between two adjacent towers is supposed 
to suffer from the same wind speed. Therefore, the failure rate $\lambda_{m, l}(t)$ of the $l$ th line segment of the $m$ th transmission corridor at time $t_{i}$ is expressed as [31]

$$
\lambda_{m, l}\left(t_{i}\right)=\exp \left[11 \times \frac{v_{m, l}\left(t_{i}\right)}{v_{d, \text { line }}}-18\right] \Delta l,
$$

where $v_{m, l}\left(t_{i}\right)$ is the wind speed of the $l$ th line segment of the $m$ th transmission corridor at time $t_{i}, v_{d \text {,line }}$ is the design wind speed of the line segment, and $\Delta l$ is the length of the $l$ th line segment.

Accordingly, the cumulative failure probability of the $l$ th line segment of the $m$ th transmission corridor within the typhoon duration $T$ is given by

$$
\begin{aligned}
p_{m, l} & =1-\exp \left(-\int_{0}^{T} \lambda_{m, l} d t\right) \\
& =1-\exp \left[-\sum_{i=0}^{N-1} \int_{t_{i}}^{t_{i}+\Delta t} \lambda_{m, l}\left(t_{i}\right) d t\right], \\
& =1-\exp \left[-\sum_{i=0}^{N-1} \lambda_{m, l}\left(t_{i}\right) \Delta t\right]
\end{aligned}
$$

Since the transmission towers and line segments are connected in series, the failure of a single tower or line segment can trip a transmission corridor. Their failures are, however, considered to be independent of the adjacent ones. According to the failure probability of the series system, the equivalent failure probability of the $m$ th transmission corridor is assumed to be equal to the aggregated failure probability of all towers and all line segments, which can be obtained by:

$$
p_{m}=1-\prod_{1}^{K}\left(1-p_{m, k}\right) \prod_{1}^{L}\left(1-p_{m, l}\right),
$$

where $K$ represents the total number of towers across the $m$ th transmission corridor, and $L$ represents the total number of line segments across the $m$ th transmission corridor.

\section{Resilience Assessment Model of a Transmission System}

Analysis of spatiotemporal cumulative failure probabilities of the transmission corridors can enable planners to determine the ones that are likely to be damaged by a typhoon, i.e. the weak points of the system. In this section, a resilience assessment model is developed to quantify the system resilience and reveal the weak points of the system. From the system perspective, the resilience assessment model can anticipate the expected typhoon-induced load shedding during the planning time period. If the results are higher than a preset limit, it means that the planning system is not resilient enough to survive typhoon strikes. Thereafter, from the perspective of the system components, the resilience assessment model can further identify vulnerable transmission corridors that are the most likely to be severely affected.

\section{1) System Resilience Assessment}

In coastal areas, typhoons may occur every year. So, planners have to take into account various potential typhoon disasters within the planning period. The landing sites, paths, and intensities of such typhoons are, however, likely to be different for each scenario. In addition, each typhoon disaster may yield different failure scenarios and result in different load shedding. To this end, the system resilience index $R_{s y s}$ is defined as the expected load shedding of the enumerated failure scenarios caused by various potential typhoon disasters throughout the planning period. It can help planners to determine whether the planning scheme is resilient enough against typhoon disasters and can be expressed as

$$
R_{s y s}=\sum_{w=1}^{W} P_{w} E\left[Q_{w}\right]
$$

where $P_{w}$ is the probability of typhoon $w, W$ is the number of various potential typhoon disasters, $E[]$ is the expected value of load shedding yielded by typhoon $w$.

\section{2) Component Resilience Assessment}

It is of vital importance to effectively expand and upgrade the planning system in order to enhance its resilience against typhoon disasters. However, it is too costly to strengthen all the transmission corridors. Therefore, the component index is developed to determine the vulnerable corridors that may cause severe load shedding when a typhoon strikes. Generally, the component resilience index of the $m$ th transmission corridor can be obtained by

$$
R_{m}=R_{s y s}-\left.R_{s y s}\right|_{p_{w, m}=0},
$$

where $R_{s y s} \mid p_{w, m}=0$ is the system resilience under the assumption that the $m$ th transmission corridor will never fail under any typhoon disasters.

This index reflects the increment of system resilience caused by the failed component. A larger value of $R_{m}$ indicates that the failure of the component $m$ is more consequential than other components, thus making it more critical to overall resilience of the system. Once the weak points are determined, multiple enhancement measures can be conducted to reduce the vulnerability of the planning system against typhoons.

\section{Algorithm Implementation}

From the long-term planning view, constructing a resilient transmission system can be achieved via the proposed framework as shown in Fig. 2. Combinatorial enumeration method [32] is implemented in the probabilistic typhoon model to yield various potential typhoon disasters, and the failure probability of transmission corridors under each typhoon can be further obtained through the spatiotemporal fragility model. In the resilience assessment model, the IISE method [30] is embedded to efficiently compute of the system and the component indices. It is worth noting that the adoption of other methods for solving each model does not affect the applicability of the proposed framework.

\section{1) Combinatorial Enumeration Method}

In this paper, the combinatorial enumeration method is used to generate various potential typhoon disaster scenarios. According to the empirical estimates from [27], the original pressure difference between the center and the periphery of the typhoon is supposed to obey a lognormal probability distribution, and so is the translational speed. The motion direction is supposed to obey a binormal probability distribution, while the landing site along the coast is supposed to obey a uniform probability distribution. Note that the 
adoption of other distributions does not affect the applicability of the proposed framework.

The probability density function of each key parameter is divided into several equal portions. A group of key parameters can be enumerated in each segmented interval, and a simulated typhoon disaster can be generated accordingly. Take the original pressure difference $\triangle H_{0}$ as an example. The original pressure difference probability density function is divided into $C$ equal portions and the segmented interval is $c_{H} . \triangle H_{0, w}$ is the original pressure difference under typhoon $w$, and its occurrence probability can be obtained via

$$
P_{r}\left(\Delta H_{0, w}\right)=\int_{\Delta H_{0, w}-c_{H} / 2}^{\Delta H_{0, w}+c_{H} / 2} f\left(\Delta H_{0}\right) d \Delta H_{0},
$$

where $P_{r}()$ is the probability of each parameter, and $c$ is the length of each portion.

Similarly, the occurrence probabilities of the translational speed $v_{T, w}$, the motion direction $\theta_{w}$ and the landing site along the coast $\left(x_{0, w}, y_{0, w}\right)$ of a typhoon $w$ can also be enumerated. The occurrence probability of a typhoon $w$ in the given region can be further obtained by (19), as shown in Fig. 4.

$$
P_{w}=P_{r}\left(\Delta H_{0, w}\right) P_{r}\left(v_{T, w}\right) P_{r}\left(\theta_{w}\right) P_{r}\left(x_{0, w}, y_{0, w}\right),
$$

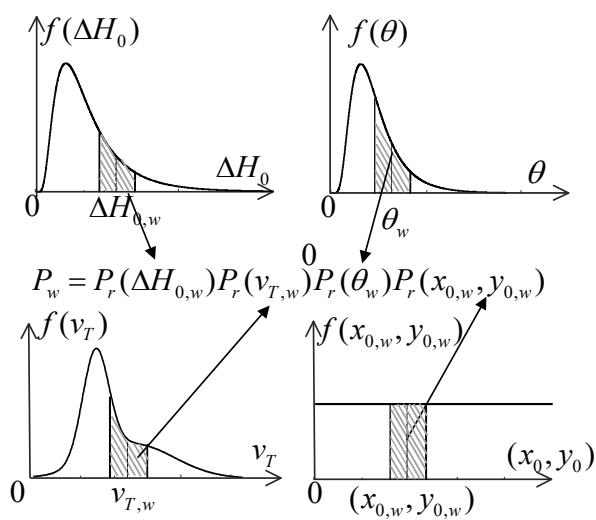

Fig. 4. The occurrence probability of typhoon $w$.

By substituting each set of key parameters into the typhoon wind field model from (8) to (13) the wind speed at any point in the typhoon-affected area can be obtained.

Then the cumulative failure probability of each corridor during the typhoon period can be further obtained from the spatiotemporal fragility model.

\section{2) IISE Method}

To balance the computational efficiency and accuracy of the resilience indices, the IISE method, developed in our previous works [33]-[35], is employed in the resilience assessment model. Considering $J$ orders failure scenarios in each typhoon, the system resilience indices based on the IISE method are obtained via

$$
R_{s y s}=\sum_{w=1}^{W} P_{w} \sum_{j=1}^{J} \sum_{s \in \Omega_{A}^{j}}\left(\prod_{i \in s} p_{w, i}\right) \Delta I_{w, s},
$$

where $\Omega_{A}^{j}$ is the $j$ order subset of $A$ and $s$ is a failure scenario denoted by a set of corresponding failed transmission corridors, $J$ is the default order of enumerated failure scenario, $p_{w, i}$ is the failure probability of the $i$ th corridor under typhoon $w$, and $\Delta I_{w, s}$ is the impact-increment of load shedding of the failure scenario $s$ under the typhoon $w$. Note that $p_{w, i}$ is obtained using the spatiotemporal fragility model in the previous subsection.

And by the same way, the component resilience indices based on the IISE method are obtained via

$$
\begin{aligned}
R_{m} & =\sum_{w=1}^{W} P_{w}\left[\sum_{j=1}^{J} \sum_{s \in \Omega_{A}^{j}}\left(\prod_{i \in s} p_{w, i}\right) \Delta I_{w, s}-\sum_{j=1}^{J} \sum_{\substack{S_{0} \in \Omega_{A}^{j} \\
m \notin s_{0}}}\left(\prod_{i \in s_{0}} p_{w, i}\right) \Delta I_{w, s_{0}}\right] \\
& =\sum_{w=1}^{W} P_{w} \sum_{j=1}^{J}\left[p_{w, m} \sum_{\substack{s_{m} \in \Omega_{A}^{j} \\
m \in s_{m}}}\left(\prod_{i \in s_{m}} p_{w, i}\right) \Delta I_{w, s_{m}}\right]
\end{aligned}
$$

where $s_{0}$ is a failure scenario that excludes the $m$ th transmission corridor, and $p_{w, m}$ is the cumulative failure probability of the $m$ th transmission corridor under typhoon $w . s_{m}$ is a failure scenario denoted by a set of corresponding failed transmission corridors, including the $m$ th transmission corridor.

With the assessment outcomes obtained from our proposed framework, corresponding resilience enhancement measures can be conducted, such as building additional transmission corridors to enable redundant transmission routes for the vulnerable corridors, updating the vulnerable corridors with more robust materials, among others [4].

\section{3) Resilience Assessment Process}

The proposed planning-oriented resilience assessment framework can not only assess whether the planning scheme makes the transmission system resilient enough against typhoon disasters, but also can guide the resilience enhancement measures aiming at the weak link. The application process of the proposed framework is shown in Fig. 5.

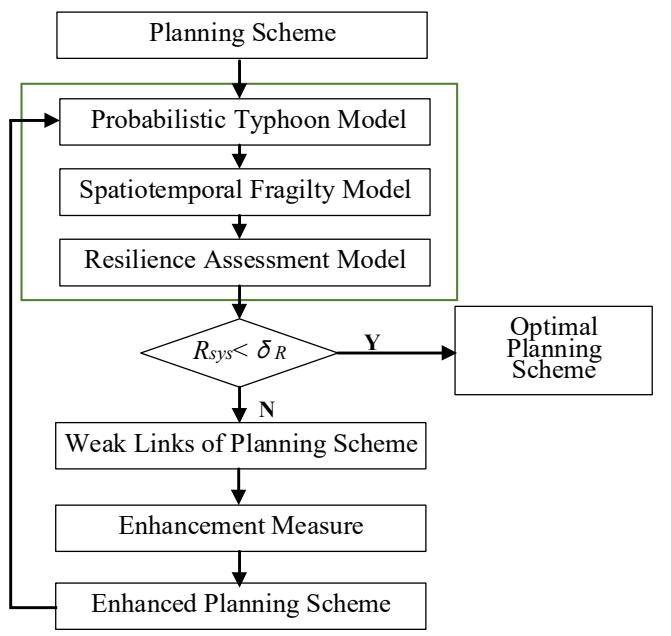

Fig. 5. The flow chart of resilience assessment under typhoon disasters

The system resilience of the planning transmission system can be obtained through the proposed framework. If the planning scheme makes the system resilient enough against typhoon disasters by comparing the preset threshold of system resilience, the planning scheme can be a candidate. On the contrary, if the planning scheme does not make the system more resilient, the enhancement measures, such as expanding or upgrading the vulnerable transmission corridors, can be 
conducted to enhance the weak links. The corresponding enhanced planning scheme can be backtracked to the planningoriented resilience assessment framework to re-assess its resilience until the system is resilient enough against typhoon disasters.

\section{CASE STUdies}

\section{A. Case Scenario}

The IEEE RTS-79 is employed to testify the practicality and effectivity of the proposed framework and method. It consists of 32 overhead transmission corridors and the total load capacity is $2850 \mathrm{MW}$. System details can be found in [36]. The transformers and cables generally have high structural reliability against typhoons, so only the transmission corridor damages are considered.

The coastal areas of Guangdong province are often affected by typhoons. We attach the IEEE RTS-79 test system on the geographic map of Guangdong province. Transmission towers are assumed to stand every 500 meters along the transmission corridors. The transmission lines are divided into several segments with a length of 500 meters. According to [37], the locations of all line segments and towers can be estimated. The wiring and location schematic diagrams of the system are shown in Fig. 6. The yellow cross indicates the location of each bus, and the blue curve indicates the coastline of Guangdong province. The simulation time interval is set to 1 hour.
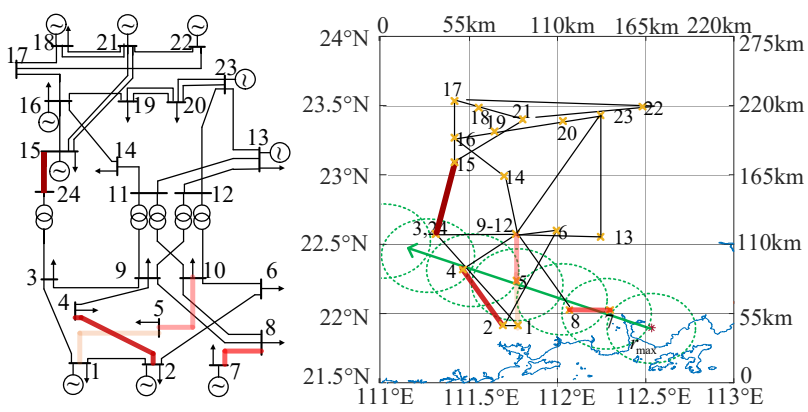

Fig. 6. The wiring schematic diagram and the location schematic diagram.

\section{B. Resilience Assessment under Typhoon Mangkhut}

Before considering the probabilistic feature of various typhoons, an actual typhoon, Mangkhut, is conducted to illustrate the calculation process. The original central pressure difference is $58 \mathrm{hPa}$, the translational speed is $30 \mathrm{~km} / \mathrm{h}$, the motion direction is west-northwest and the landing site is $21.9^{\circ}$ $\mathrm{N} / 112.5^{\circ} \mathrm{E}$ [38]. The path of typhoon motion is shown by the green dotted arrow in Fig 5. Green circles represent the radius of maximum wind speed for each hour. Five corridors of different sites are randomly selected as examples to demonstrate the proposed method. They are Corridor-2 (bus 1 to 3), Corridor-8 (bus 4 to 9), Corridor-12 (bus 8 to 9), Corridor20 (bus 12 to 13) and Corridor-27 (bus 15 to 24). Those corridors contain both a series of line segments and towers. The wind speeds in the middle of these corridors are shown in Fig. 7 (a). It can be seen that the wind speed of different corridors varies with time as the typhoon moves across the system.

Take Corridor-2 as an example. According to (4), in the early stage after typhoon landing, the wind speed increases continuously with the decreasing distance between Corridor-2 and the typhoon center. However, when the distance is less than the radius of maximum wind, the wind speed begins to decrease. As the typhoon continues to move, the distance begins to increase again, but it is still less than the radius of maximum wind, yielding decreased wind speed. When the distance continues to increase to the radius of maximum wind, the wind speed begins to decrease again and further decrease as the typhoon moves away.

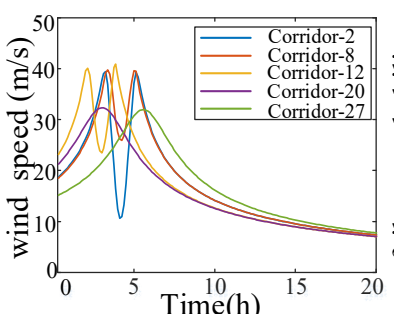

(a) wind speed

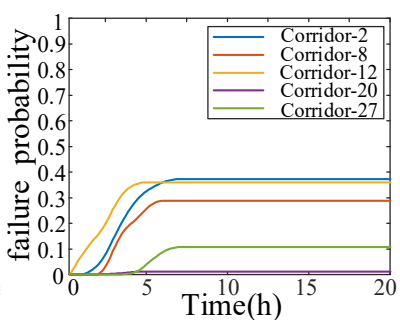

(b) failure probability
Fig. 6. The wind speed and the cumulative failure probability of corridors.

The cumulative failure probabilities of those corridors are also shown in Fig. 7 (b) and they increase with time. This is because in the early stage of typhoon landing, the wind speed increases sharply. Similarly, the cumulative failure probability of each transmission corridor also has a similar behavior. With the movement and attenuation of the typhoon, the wind speed decreases, and the cumulative failure probability still increases very slowly. The cumulative failure probability of the rest corridors can be obtained in the same way.

The corresponding failure scenarios set for the resilience assessment can then be established. The default order of the enumerated failure scenario is set to 2 for illustration purposes. Monte Carlo simulation (MCS) method [39] is used as a benchmark method and the convergence criterion is set to $10^{7}$ sampled failure scenarios. SE method is implemented for comparison. Calculation results are listed in Table I, where IISE2 and SE2 represent the result obtained by the IISE and the SE methods, which are based on a $2^{\text {th }}$ order of enumerated failure scenarios. It can be seen from Table I that the IISE method is more accurate than the SE method with nearly the same computation time. So, in the following assessment process, the IISE method will be adopted.

TABLE I

THE SYSTEM RESILIENCE INDICES UNDER TYPHOON MANGKHUT

\begin{tabular}{cccc}
\hline \hline Methods & $\begin{array}{c}E\left[\boldsymbol{Q}_{\boldsymbol{w}}\right] \\
(\text { MWh/oc.) })\end{array}$ & Error & $\begin{array}{c}\text { Time } \\
(\mathrm{s})\end{array}$ \\
\hline MCS & 34.03 & - & $1.613 \mathrm{E} 5$ \\
SE2 & 2.079 & $93.89 \%$ & $2.702 \mathrm{E} 1$ \\
IISE2 & 33.91 & $0.353 \%$ & $3.165 \mathrm{E} 1$ \\
\hline
\end{tabular}

Subsequently, a component-level evaluation is conducted to determine the weak corridors that are more vulnerable to this typhoon. Recall that the cumulative failure probability of each transmission corridor and the impact-increment of each failure scenario have already been obtained during the calculation process of the system resilience assessment. The component resilience indices can be directly obtained without any optimal power flow calculation and the results are listed in Table II. 
These vulnerable corridors are also marked in red in Fig.5. The darker the red line is, the more fragile the corridor is. It can be found that the index of Corridor-27 is larger than the others, indicating that its failure will result in more severe consequences under typhoon Mangkhut.

TABLE II

THE COMPONENT RESILIENCE INDICES

\begin{tabular}{cc}
\hline \hline Index & $\begin{array}{c}\text { Result } \\
\text { (MWh/occ.) }\end{array}$ \\
\hline $\boldsymbol{R}_{\mathbf{2 7}}$ (Bus 15 to 24) & 10.60 \\
$\boldsymbol{R}_{\mathbf{4}}$ (Bus 2 to 4) & 7.476 \\
$\boldsymbol{R}_{\mathbf{1 1}}$ (Bus 7 to 8) & 7.002 \\
$\boldsymbol{R}_{\mathbf{9}}$ (Bus 5 to 10) & 6.374 \\
$\boldsymbol{R}_{\mathbf{3}}$ (Bus 1 to 5) & 6.264 \\
\hline \hline
\end{tabular}

\section{Resilience Assessment under Potential Typhoon Disasters}

Using historical data of typhoons, the probability density function of each key parameter in the given region is obtained. Such functions are then employed for the probabilistic typhoon model to calculate the occurrence probability of various potential typhoon disasters via combinatorial enumeration. Each set of key parameters only generates a certain typhoon. The key typhoon parameters of this region are fitted according to the tracking data of the tropical cyclone in the Northwest Pacific provided by the Chinese Meteorological Administrator for the period from 1949 to 2011, as well as the 2000 geographical coordinate data of coastline in Guangzhou province [27]. The probability density function of each key parameter is divided into ten equal portions, and the combinatorial enumeration number is 10000 . The resilience indices can be obtained by repeating the calculation process under each generated typhoon. The calculation result of the system resilience index is listed in Tab. III (first row).

TABLE III

THE COMPONENT RESILIENCE INDICES

\begin{tabular}{cc}
\hline \hline Index & $\begin{array}{c}\text { Result } \\
(\text { MWh })\end{array}$ \\
\hline $\boldsymbol{R}_{\text {sys }}$ & 1.984 \\
$\boldsymbol{R}_{\mathbf{1 1}}($ Bus 7 to 8) & 0.925 \\
$\boldsymbol{R}_{\mathbf{2 7}}$ (Bus 15 to 24) & 0.531 \\
$\boldsymbol{R}_{\mathbf{1 3}}$ (Bus 8 to 10) & 0.513 \\
$\boldsymbol{R}_{\mathbf{1 2}}$ (Bus 8 to 9) & 0.319 \\
$\boldsymbol{R}_{\mathbf{4}}$ (Bus 2 to 4) & 0.205 \\
\hline \hline
\end{tabular}

For regions that are frequently struck by typhoons, how to expand, or upgrade them in the planning period is an issue worthy of further study. Given that the system resilience index does not indicate which corridors should be expanded or upgraded, it is necessary to calculate the component resilience indices to obtain the vulnerable transmission corridors. According to the proposed component resilience indices, the occurrence probability of each typhoon, the cumulative failure probability of each transmission corridor and the impactincrement of each failure scenario have been obtained during the calculation process of the system resilience index. The results of the component resilience index and the five most vulnerable corridors are listed in Table III (from the second to the sixth row). From this data, it is deducible that Corridor-11 is the most vulnerable to typhoon disasters. The results can further provide a reference for enhancement strategies to increase the system resilience to typhoons.

To validate the effect of the component resilience indices on the implementation of resilience enhancement measures, two different measures are considered: 1) Expansion: adding redundant corridor parallel to the vulnerable transmission; and 2) Upgrading: building the transmission corridors with more robust materials. In the former, an additional corridor is built in parallel with each vulnerable corridor, while in the latter, each original corridor is upgraded with more robust material. Upgrading a corridor is achieved by increasing the designed wind speed of each line segment and tower. For the sake of comparison, the percentage of the system index reduction is used to evaluate the effects of enhancement under each scheme.

TABLE IV

THE ENHANCED SYSTEM RESILIENCE INDICES

\begin{tabular}{ccccc}
\hline \hline Corridor & $\begin{array}{c}\boldsymbol{S}_{E x p} \\
\text { (MWh) }\end{array}$ & $\triangle \boldsymbol{S}_{\text {Exp }}$ & $\begin{array}{c}\boldsymbol{S}_{\text {Upg }} \\
\text { (MWh) }\end{array}$ & $\triangle \boldsymbol{S}_{\text {Upg }}$ \\
\hline $\boldsymbol{R}_{\mathbf{1 1}}$ (Bus 7 to 8) & 0.995 & $49.85 \%$ & 1.106 & $44.25 \%$ \\
$\boldsymbol{R}_{\mathbf{1 3}}$ (Bus 8 to 10) & 1.113 & $43.90 \%$ & 1.476 & $25.60 \%$ \\
$\boldsymbol{R}_{\mathbf{2 7}}$ (Bus 15 to 24) & 1.298 & $34.58 \%$ & 1.503 & $24.24 \%$ \\
$\boldsymbol{R}_{\mathbf{1 2}}$ (Bus 8 to 9) & 1.487 & $25.05 \%$ & 1.684 & $15.12 \%$ \\
$\boldsymbol{R}_{\mathbf{4}}$ (Bus 2 to 4) & 1.731 & $12.75 \%$ & 1.791 & $9.73 \%$ \\
\hline \hline
\end{tabular}

The system resilience indices of the enhancement schemes are listed in Table IV. Here, $S_{E x p}$ and $\triangle S_{E x p}$ denote the resilience and the corresponding enhancement effect of the expanding, while $S_{U p g}$ and $\triangle S_{U p g}$ denote the resilience and the corresponding enhancement effect of the upgrading. The ranking result of each enhancement scheme is consistent with the ranking of the component resilience indices. In the case of limited resources, increasing redundancy in the most vulnerable corridor is a more effective action than upgrading them. These results illustrate the role of the component resilience indices in determining the vulnerable corridors and guiding the planning schemes toward enhancing the resilience of the transmission systems.

\section{CONCLUSION AND FUTURE WORK}

This paper proposes a planning-oriented resilience assessment framework. Firstly, a probabilistic typhoon model is proposed, which depicts the uncertain features of typhoons by the probability distribution of key parameters. This allows us to generate a series of typhoon disasters and their occurrence probabilities. Secondly, a spatiotemporal fragility model of the transmission corridors is constructed considering the movement and attenuation of the typhoon. Those models quantify the relationship between the failure probability of each transmission corridor and the wind speed from both time and space scales. Finally, a resilience assessment model is developed to quantify the resilience of the transmission system from the perspectives of the system and the component. Combinatorial enumeration method is introduced into the probabilistic typhoon model to generate various potential typhoon disasters, and the failure probability of transmission corridors under each typhoon can be further obtained via spatiotemporal fragility model. In addition, the IISE method is embedded into the resilience assessment model to efficiently compute the system and the component indices.

Numerical results verify the accuracy and efficiency of the 
proposed framework. The outcomes can also provide insights into potential resilience enhancement measures against future similar or unforeseen typhoon disasters. With the proposed method, resilient expansion and upgrade of the planning transmission system can be conducted effectively and economically. In addition, the proposed method can be easily extended to deal with other extreme events.

The resilience enhancement measures in this paper, such as expanding or upgrading the vulnerable transmission corridors, belong to the research category of prevention control in long time scale. However, the restoration in short-time scale is also an important way to enhance system resilience. In the future, we will extend our research to address both prevention and restoration enhancement measures. Furthermore, the impacts of interactions between adjacent components are not considered in this paper. Since the transmission towers and line segments are connected in series, the failure of a single tower or line segment may trip a transmission corridor. The interactions can be considered by a joint probability distribution of dependent line. We will extend our work to address that.

\section{APPENDIX}

\section{A. Probability Density Functions of Key Parameters}

According to the empirical estimates from [28], the original pressure difference between the center is supposed to obey a lognormal probability distribution as follows:

$$
f(\Delta H)=\frac{1}{\Delta H \sigma \sqrt{2 \pi}} \exp \left(-\frac{(\ln \Delta H-\mu)^{2}}{2 \sigma^{2}}\right),
$$

where $\mu=2.9001, \sigma=0.6274$.

The translational speed of the typhoon is supposed to obey a lognormal probability distribution as follows:

$$
f\left(v_{T}\right)=\frac{1}{v_{T} \sigma \sqrt{2 \pi}} \exp \left(-\frac{\left(\ln v_{T}-\mu\right)^{2}}{2 \sigma^{2}}\right)
$$

where $\mu=2.6680, \sigma=0.5185$.

The motion direction is assumed to obey a binormal probability distribution as follows

$$
f(\theta)=\frac{\alpha}{\sigma_{1} \sqrt{2 \pi}} \exp \left[-\frac{\left(\theta-\mu_{1}\right)^{2}}{2 \sigma_{1}^{2}}\right]+\frac{1-\alpha}{\sigma_{2} \sqrt{2 \pi}} \exp \left[-\frac{\left(\theta-\mu_{2}\right)^{2}}{2 \sigma_{2}^{2}}\right]
$$

where $\mu_{1}=-73.3392, \quad \mu_{2}=-7.2084, \quad \sigma_{1}=22.5891, \quad \sigma_{2}=70.3532$, $\alpha=0.5035$.

The landing site along the coast is assumed to obey a uniform probability distribution as follows:

$$
f\left(x_{0}\right)=\frac{1}{b_{x}-a_{x}}, f\left(y_{0}\right)=\frac{1}{b_{y}-a_{y}}
$$

where $\left(a_{x}, a_{y}\right)$ and $\left(b_{x}, b_{y}\right)$ are the coordinates of the starting and ending points of the coastline of the studied areas, respectively.

\section{B. The Buses Involved with The Corridor}

Table $\mathrm{V}$ shows the buses involved with each corridor. It is worth mentioning that corridors 25 and 26, 32 and 33, 34 and 35,36 and 37 are double circuit transmission ones. The failures of double circuit transmission corridors are considered to be independent in our paper.
TABLE V

THE Bus INVOLVED WITH THE CORRIDOR

\begin{tabular}{cccccc}
\hline \hline Corridor & From Bus & To Bus & \multicolumn{2}{c}{ Corridor From Bus } & To Bus \\
\hline 1 & 1 & 2 & 18 & 11 & 13 \\
2 & 1 & 3 & 19 & 11 & 14 \\
3 & 1 & 5 & 20 & 12 & 13 \\
4 & 2 & 4 & 21 & 12 & 23 \\
5 & 2 & 6 & 22 & 13 & 23 \\
6 & 3 & 9 & 23 & 14 & 16 \\
7 & 3 & 24 & 24 & 15 & 16 \\
8 & 4 & 9 & $25+26$ & 15 & 21 \\
9 & 5 & 10 & 27 & 15 & 24 \\
10 & 6 & 10 & 28 & 16 & 17 \\
11 & 7 & 8 & 29 & 16 & 19 \\
12 & 8 & 9 & 30 & 17 & 18 \\
13 & 8 & 10 & 31 & 17 & 22 \\
14 & 9 & 11 & $32+33$ & 18 & 21 \\
15 & 9 & 12 & $34+35$ & 19 & 20 \\
16 & 10 & 11 & $36+37$ & 20 & 23 \\
17 & 10 & 12 & 38 & 21 & 22 \\
\hline \hline
\end{tabular}

\section{REFERENCES}

[1] Executive Office of the President, "Economic benefits of increasing electric grid resilience to weather outages," Tech. Rep., USA, Aug. 2013.

[2] R. J. Campbell, "Weather-Related Power Outages and Electric System Resiliency," Congressional Res. Service Rep., Aug. 2012.

[3] J. Lu, M. Zeng, X. Zeng, Z. Fang and J. Yuan, "Analysis of ice-covering characteristics of China Hunan power grid," IEEE Trans. Ind. Appl, vol. 51, no. 3, pp. 1997-2002, May-June, 2015.

[4] M. Panteli and P. Mancarella, "The grid: stronger, bigger, smarter? Presenting a conceptual framework of power system resilience," IEEE Power Energy Mag., vol. 13, no. 3, pp. 58-66, May-Jun. 2015.

[5] R. Rocchetta, E. Zio and E. Patelli, "A power-flow emulator approach for resilience assessment of repairable power grids subject to weather-induced failures and data deficiency". Appl. Energy, vol. 210, pp. 339-350, Jan. 2018

[6] National Research Council, "The Resilience of the Electric Power Delivery System in Response to Terrorism and Natural Disasters: Summary of a Workshop," Washington, DC: The National Academies Press, 2013.

[7] Y. Wang, C. Chen, J. Wang, and R. Baldick, "Research on resilience of power systems under natural disasters-a review," IEEE Trans. Power Syst., vol. 31, no. 2, pp. 1604-1613, Mar. 2016

[8] Y. Fang, N. Pedroni and E. Zio, "Resilience-based component importance measures for critical infrastructure network systems," IEEE Trans. Reliab., vol. 65, no. 2, pp. 502-512, June. 2016.

[9] M. H. Amirioun, F. Aminifar, H. Lesani, and M. Shahidehpour, "Metrics and quantitative work for assessing microgrid resilience against windstorms," Int. J. Electr. Power Energy Syst., vol. 104, pp. 716-723, Jan. 2019.

[10] M. Batts, L. Russell and E. Simiu, "Hurricane wind speeds in the United States,” J. Struct. Division, vol. 106, no. 10, pp. 2001-2016, Jan. 1980.

[11] L. Shapiro, "The asymmetric boundary layer flow under a translating hurricane,” J. Atmo. Sci., vol. 40, no. 8, pp. 1984-1998, Jul. 1983.

[12] Y. Meng, M. Matsui and K. Hibi, "An analytical model for simulation of the wind field in a typhoon boundary layer," J. Wind Eng. \& Ind. Aerody., vol. 56, no. 2, pp. 291-310, May. 1995.

[13] Y. Liu and C. Singh. "A Methodology for Evaluation of Hurricane Impact on Composite Power System Reliability," IEEE Trans. Power Syst., vol. 26, no. 1, pp. 145-152, Feb. 2011.

[14] W. Li, J. Zhou and X. Xiong. "Fuzzy models of overhead power line weather-related outages,” IEEE Trans. Power Syst., vol. 23, no. 3, pp. 1529-1531, Aug. 2008.

[15] H. Liu, R. Davidson, D. Rosowsky and J. Stedinger, "Negativebinomial regression of electric power outages in hurricanes," J. Inf. Syst, vol. 11, no. 4 , pp. 258-267, Dec. 2005.

[16] S. Han, S. Guikema, S. Quiring, K. Lee, D. Rosowsky and R. Davidson, "Estimating the spatial distribution of power outages during hurricanes in the gulf coast region," Reliab. Eng. Syst. Saf., vol. 94, no. 2, pp. 199210, Feb. 2009.

[17] M. Panteli and P. Mancarella, "Operational resilience assessment of power systems under extreme weather and loading conditions", IEEE PES General Meeting 2015, Denver, USA, 26-30, Jul. 2015. 
[18] M. Panteli, D. N. Trakas, P. Mancarella, and N. D. Hatziargyriou, "Boosting the Power Grid Resilience to Extreme Weather Events Using Defensive Islanding," IEEE Trans. Smart Grid, vol. 7, no. 6, pp. 29132922, Nov. 2016.

[19] M. Panteli, C. Pickering, S. Wilkinson, R. Dawson and P. Mancarella. "Power system resilience to extreme weather: fragility modeling, probabilistic impact assessment and adaptation measures", IEEE Trans. Power Syst., vol. 32, no. 5, pp. 3747-3757, Sept. 2017.

[20] C. Wang, Y. Hou, F. Qiu, S. Lei, and K. Liu, "Resilience enhancement with sequentially proactive operation strategies," IEEE Trans. Power Syst., vol. 32, no. 4, pp. 2847-2857, Jul. 2017.

[21] G. Li, P. Zhang, P. Luh, W. Li, Z. Bie, C. Serna and Zhibing Zhao., "Risk analysis for distribution systems in the northeast U.S. under wind storms," IEEE Trans. Power Syst., vol. 29, no. 2, pp. 889-898, Mar. 2014.

[22] M. Bruneau, S. Chang, R. Eguchi, G. Lee, T. O’Rourke, A. Reinhorn, M. Shinozuka, K. Tierney, W. Wallace and D. Winterfeldt, "A Framework to Quantitatively Assess and Enhance the Seismic Resilience of Communities," Earthq. Spectra, vol.19 no.4, pp. 733-752, Nov. 2003.

[23] M. Panteli, P. Mancarella, D. Trakas, E. Kyriakides and N. Hatziargyriou, "Metrics and quantification of operational and infrastructure resilience in power systems", IEEE Trans. Power Syst., vol. 32, no. 6, pp. 47324742, Nov. 2017

[24] S. Espinoza, M. Panteli, P. Mancarella, and H. Rudnick, "Multi-phase assessment and adaptation of power systems resilience to natural hazards," Electr. Power Syst. Res., vol. 136, pp. 352-361, Jul. 2016.

[25] M. H. Amirioun, F. Aminifar, and H. Lesani, "Resilience-oriented proactive management of microgrids against windstorms," IEEE Trans. Power Syst., vol. 33, no. 4, pp. 4275-4284, July. 2018.

[26] B. Johnson, V. Chalishazar, E. Cotilla-Sanchez and T. Brekken, "A Monte Carlo methodology for earthquake impact analysis on the electrical grid,". Electric Power Syst. Research, no. 184, pp. 106332, July. 2020.

[27] M. Chen, "The wind power reliability modeling research considering the effects of typhoon," M.S. thesis, Dept. Power System and its Automation, Guangxi Univ., Guangxi, China, 2014

[28] P. Vickery, P. Skerlj and L. Twisdale, "Simulation of hurricane risk in the U.S. using empirical track mode," J. Struct. Eng., vol. 126, no. 10, pp. 1222-1237, Oct. 2000

[29] Y. Wang and D. Rosowsky, "Joint distribution model for prediction of hurricane wind speed and size", Struct Saf., vol. 35, pp. 40-51, Jan. 2012.

[30] Y. Chen, S. Wang, B. Chen, T. Xu, C. Yu and J. Yu, "Evaluation of the Failure Probability of Power Transmission Corridors During Typhoons Using Digital Elevation Information," Power Syst. Tech., vol. 42, no. 7, pp. 2295-2302, Jul. 2018

[31] X. Song, Z. Wang, D. Gan, and J. Qiu, "Transient stability risk assessment of power grid under typhoon weather," Power Syst. Prot. Control, vol. 40, no. 24, pp. 1-8, Dec. 2012.

[32] J. Zhao and D. He, "Research and application of enumeration combination algorithm," Sci. Tech. Inf., vol. 10, pp. 74-75, 2009.

[33] K. Hou, H. Jia, X. Yu, L. Zhu, X. Xu and X. Li, "An Impact IncrementBased State Enumeration Reliability Assessment Approach and Its Application in Transmission Systems," IEEE PES General Meeting, Boston, MA, USA, Jul. 2016.

[34] K. Hou, H. Jia, X. Li, X. Xu, Y Mu, J. Tao and X. Yu, "Impact-increment based decoupled reliability assessment approach for composite generation and transmission systems," IET Generation, Transm. Distribution, vol. 12, no. 3, pp. 586-595, Feb. 2018.

[35] X. Liu, K. Hou, H. Jia, J. Zhao, L. Mili, Y. Mu, J. Rim, Y. Lei, "A resilience assessment approach for power system from perspectives of system and component levels," Int. J. Electr. Power Energy Syst., vol. 118, pp. 105837, Jun., 2020.

[36] IEEE RTS Task Force of APM Subcommittee, "IEEE reliability test system," IEEE Trans. Power Appa. Syst., vol. PAS-98, no. 6, pp. 2047 2054, Nov-Dec. 1979.

[37] R. Allan and R. Billinton, "The IEEE Reliability Test System 1996. A Report by the Reliability Test System Task Force," IEEE Trans. Power Syst., Vol. 14, No. 3, pp. 1010-1020, Aug. 1999.

[38] Weather China [Online]. Available: http://typhoon.weather.com.cn/

[39] D. Lieber, A. Nemirovskii and R. Y. Rubinstein, "A fast Monte Carlo method for evaluating reliability indexes," IEEE Trans. Reliab., vol. 48 , no. 3, pp. 256-261, Sept. 1999.

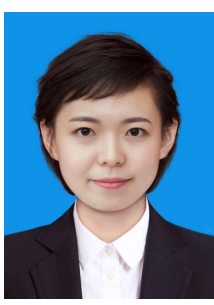

Xiaonan Liu received the B.S. degree in power system and its automation form Shanghai University of Electric Power, Shenyang, China, in 2011. She received the M.S. degree in electrical engineering from Shenyang Institute of Technology, Shenyang, China, in 2015. She is currently working toward the Ph.D. degree in the Electrical Engineering Department, Tianjin University, Tianjin, China.

Her research interests include reliability and resilience assessment of power systems.

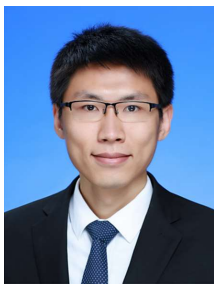

Kai Hou (M'16) received the Ph.D. degree in electrical engineering from Tianjin University, Tianjin, China, in 2016. He is currently an Associate Professor with the Electrical Engineering Department, Tianjin University.

His research interests include reliability and risk assessments of power systems, integrated energy systems, and smart grids.

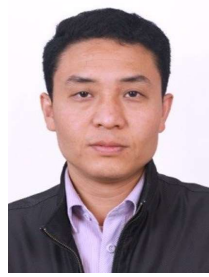

Hongjie Jia (M'04, SM'17) received the B.S., M.S. and $\mathrm{Ph} . \mathrm{D}$. degree in electrical engineering from Tianjin University, Tianjin, China, in 1996, 1999 and 2001, respectively. He is currently a Professor with Tianjin University.

His research interests include power reliability assessment, stability analysis and control, distribution network planning and automation, and smart grids.

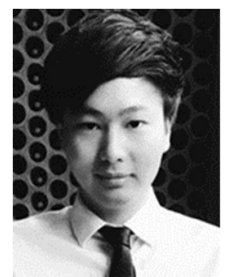

Junbo Zhao (SM'19) received the Ph.D. degree from the Department of Electrical and Computer Engineering, Virginia Tech, Blacksburg, VA, USA, in 2018. He is currently an Assistant Professor at Mississippi State University, Starkville, MS, USA

His research interests are cyber-physical power system modeling, estimation, security, dynamics and stability, uncertainty quantification, robust statistical signal processing and machine learning for smart grid.

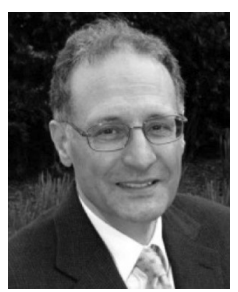

Lamine Mili (LF'17) received the B.S. degree from the Swiss Federal Institute of Technology, Lausanne, Switzerland, in 1976, and the Ph.D. degree from the University of Liege, Liege, Belgium, in 1987. He is currently a Professor of Electrical and Computer Engineering with Virginia Tech, Blacksburg, VA, USA.

His research interests include power system planning for enhanced resiliency and sustainability, risk management of complex systems to catastrophic failures, robust estimation and control, nonlinear dynamics and bifurcation theory.

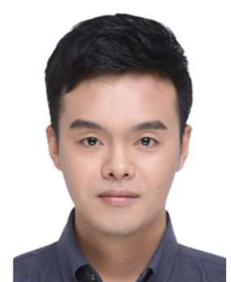

Xiaolong Jin (M'16) received the B.S., M.S. and Ph.D. degree in electrical engineering from Tianjin University, China in 2012, 2015 and 2018, respectively. He is currently a Postdoc researcher with Technical University of Denmark.

His research interests include energy management of multi-energy buildings and their integrations with multienergy systems, and the energy \& flexibility markets solutions.

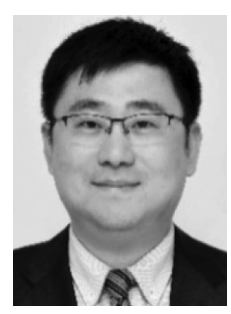

Dan Wang (M'11) received the B.S. and M.S. degrees in electric power engineering from Hohai University, Nanjing, China, in 2003 and 2006, respectively, and the $\mathrm{Ph} . \mathrm{D}$. degree in electric power engineering from Tianjin University, Tianjin, China, in 2009.

$\mathrm{He}$ became an Associate Professor at Tianjin University, in 2015. His research interests include integrated energy systems, distributed generation systems, microgrid modeling and simulation, demandside management, and power system stability analysis. 